\title{
Update on the 2+1+1 flavor QCD equation of state with HISQ
}

\section{A. Bazavov ${ }^{*, a}$, ; C. Bernard ${ }^{b}$, C. DeTar $c$, J. Foley ${ }^{c}$, Steven Gottlieb ${ }^{d}$, Urs M. Heller ${ }^{e}$ J.E. Hetrick ${ }^{f}$, J. Laiho ${ }^{g}$, L. Levkova ${ }^{c}$, J. Osborn ${ }^{h}$, R. Sugar ${ }^{i}$, D. Toussaint ${ }^{j}$, R.S. Van de Water ${ }^{k}$, and R. Zhou ${ }^{k}$}

${ }^{a}$ Department of Physics, Brookhaven National Laboratory, Upton, NY 11973, USA

${ }^{b}$ Department of Physics, Washington University, St. Louis, MO 63130, USA

${ }^{c}$ Department of Physics and Astronomy, University of Utah, Salt Lake City, UT 84112, USA

${ }^{d}$ Department of Physics, Indiana University, Bloomington, IN 47405, USA

${ }^{e}$ American Physical Society, One Research Road, Ridge, NY 11961, USA

${ }^{f}$ Physics Department, University of the Pacific, Stockton, CA 95211, USA

${ }^{g}$ Department of Physics, Syracuse University, Syracuse, NY 13244, USA

${ }^{h}$ Argonne Leadership Computing Facility, Argonne National Laboratory, Argonne, IL 60439, USA

${ }^{i}$ Department of Physics, University of California, Santa Barbara, CA 93106, USA

${ }^{j}$ Department of Physics, University of Arizona, Tucson, AZ 85721, USA

${ }^{k}$ Theoretical Physics Department, Fermi National Accelerator Laboratory, Batavia, IL 60510, USA

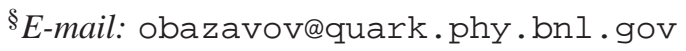

We present recent results on the QCD equation of state with $2+1+1$ flavors of highly improved staggered quarks (HISQ). We focus on three sets of ensembles with temporal extent $N_{\tau}=6,8$ and 10 , that reach up to temperatures of 967,725 and $580 \mathrm{MeV}$, respectively. The strange and charm quark masses are tuned to the physical values and the light quarks mass is set to one fifth of the strange. This corresponds to a Goldstone pion of about $300 \mathrm{MeV}$.

31st International Symposium on Lattice Field Theory LATTICE 2013

July 29 - August 3, 2013

Mainz, Germany

\footnotetext{
* Speaker.

${ }^{\dagger}$ Present address: Department of Physics and Astronomy, University of Iowa, Iowa City, IA 52245, USA
} 


\section{Introduction}

Properties of the high-temperature phase of QCD, the quark-gluon plasma, are currently a subject of investigation in ultra-relativistic heavy-ion collision experiments at RHIC (BNL), LHC (CERN) and planned future experiments FAIR (GSI) and NICA (JINR). The QCD equation of state with $2+1$ flavors of quarks has been and is being extensively studied on the lattice [1. 2] and some preliminary results for the $2+1+1$ flavor equation of state (i.e. with a dynamical charm quark) are

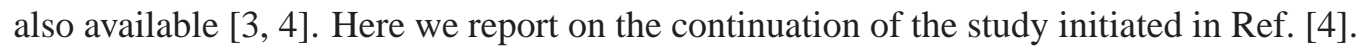

In heavy-ion experiments heavy quarks are absent in the colliding nuclei and are created at early stages of the collision. Therefore they play an important role, both theoretically and experimentally, as probes of the deconfined medium. Although the charm quark mass is on the order of $10 T_{c}\left(T_{c}=154(9) \mathrm{MeV}\right.$ being the chiral crossover temperature [5]), perturbative [6] and quenched charm lattice [7] calculations indicate that the charm contribution to the equation of state becomes non-negligible at temperatures as low as $2-3 T_{c}$. These temperatures are within reach of the heavyion program at LHC. Therefore, it seems timely to include the dynamical charm quark in $a b$ initio QCD calculations.

\section{Lattice setup}

Calculation of the equation of state on the lattice is computationally expensive because it requires subtraction of ultra-violet divergences, and both finite- and zero-temperature ensembles with large statistics are needed at every value of the gauge coupling. Therefore this study has been done along the line of constant physics (LCP) with the light quark mass set to $m_{l}=m_{s} / 5$ and makes use of a set of the existing MILC zero-temperature ensembles [8] on this LCP.

We use the tadpole one-loop improved gauge action and the highly improved staggered quark (HISQ) action [9]. The HISQ action suppresses the taste-exchange interactions present in the staggered formalism and significantly reduces the mass splittings between various pion tastes. This feature improves the approach to the continuum limit at low temperatures. It is also $O\left(a^{2}\right)$-improved, with the Naik (three-link) term, which controls scaling at high temperatures. In lattice units the charm quark mass $a m_{c} \sim O(1)$, therefore the Naik term includes a mass-dependent correction, $\varepsilon_{N}$ [9]. It is derived perturbatively to reproduce the correct charm quark dispersion relation up to $O\left(\left(a m_{c}\right)^{4}\right)$. To set the lattice spacing $a$ we use the scale $r_{1} \simeq 0.31 \mathrm{fm}$ [10]. The strange and charm quark masses are tuned to the physical values by using the $\pi, K, \eta_{c}$ and $J / \psi$ masses. The tadpole factor defined from the trace of the plaquette $u_{0}=\left\langle\operatorname{Tr} U_{p} / 3\right\rangle^{1 / 4}$ is determined during the equilibration of the zero-temperature ensembles. We used finite-temperature lattices with aspect ratio of four and temporal extent $N_{\tau}=6,8$ and 10. (In this round we did not pursue the exploratory $N_{\tau}=12$ ensembles reported in Ref. [ [ 4 , since to get a reliable signal would require significant computational resources.) The temperature is set as $T=1 /\left(a N_{\tau}\right)$.

We determined the $\beta$-functions by fitting the data to the following Ansätze. For the lattice spacing:

$$
\frac{r_{1}}{a}(\beta)=\frac{c_{r}^{(0)} f(\beta)+c_{r}^{(2)}(10 / \beta) f^{3}(\beta)}{1+d_{r}^{(2)}(10 / \beta) f^{2}(\beta)},
$$




\begin{tabular}{|l|l|l|l|l|l||l|r|}
\hline$\beta$ & $a m_{s}$ & $a m_{c}$ & $u_{0}$ & $\varepsilon_{N}$ & $a, \mathrm{fm}$ & size & $\mathrm{TU} / 10^{3}$ \\
\hline $5.400 *$ & 0.091 & 1.339 & 0.83496 & -0.7995 & 0.220 & $16^{3} \times 40$ & 5.0 \\
5.469 & 0.0928 & 1.263 & 0.838768 & -0.6905 & 0.206 & $24^{3} \times 32$ & 9.7 \\
5.541 & 0.0859 & 1.157 & 0.842646 & -0.5797 & 0.192 & $24^{3} \times 32$ & 9.7 \\
$5.60 *^{3}$ & 0.0785 & 1.080 & 0.845768 & -0.5168 & 0.181 & $16^{3} \times 48$ & 12.5 \\
5.663 & 0.0753 & 0.996 & 0.848919 & -0.4571 & 0.170 & $24^{3} \times 32$ & 9.7 \\
5.732 & 0.0697 & 0.913 & 0.852242 & -0.4024 & 0.159 & $32^{4}$ & 3.8 \\
$5.800 *$ & 0.065 & 0.838 & 0.85535 & -0.3582 & 0.151 & $16^{3} \times 48$ & 98.8 \\
5.855 & 0.0608 & 0.782 & 0.857786 & -0.3195 & 0.140 & $32^{4}$ & 12.1 \\
5.925 & 0.0561 & 0.716 & 0.860718 & -0.2784 & 0.130 & $32^{4}$ & 14.5 \\
$6.000 *$ & 0.0509 & 0.635 & 0.86372 & -0.2308 & 0.121 & $24^{3} \times 64$ & 11.4 \\
6.060 & 0.0481 & 0.603 & 0.865978 & -0.2010 & 0.113 & $32^{4}$ & 9.7 \\
6.122 & 0.0448 & 0.558 & 0.86824 & -0.1838 & 0.106 & $32^{4}$ & 9.7 \\
6.180 & 0.042 & 0.518 & 0.870236 & -0.1613 & 0.100 & $32^{4}$ & 7.8 \\
6.238 & 0.0392 & 0.482 & 0.872177 & -0.1418 & 0.094 & $32^{4}$ & 9.7 \\
$6.300 *$ & 0.037 & 0.440 & 0.874164 & -0.1204 & 0.089 & $32^{3} \times 96$ & 6.0 \\
6.530 & 0.028 & 0.338 & 0.880888 & -0.0734 & 0.070 & $36^{3} \times 48$ & 2.8 \\
$6.720 *$ & 0.024 & 0.286 & 0.885773 & -0.0533 & 0.058 & $48^{3} \times 144$ & 5.9 \\
$7.000 *$ & 0.0158 & 0.188 & 0.892186 & -0.0235 & 0.045 & $64^{3} \times 192$ & 0.7 \\
7.140 & 0.0145 & 0.172 & 0.895074 & -0.0197 & 0.039 & $64^{3} \times 72$ & 1.3 \\
7.285 & 0.0124 & 0.148 & 0.89789 & -0.0146 & 0.034 & $64^{3} \times 96$ & 0.9 \\
\hline
\end{tabular}

Table 1: The parameters of the HISQ ensembles along the $m_{l}=m_{s} / 5$ LCP: inverse gauge coupling $\beta=$ $10 / g^{2}$, input strange and charm quark masses, tadpole factor $u_{0}$, the Naik term correction $\varepsilon_{N}$, approximate lattice spacing $a$, lattice volume and the statistics in thousands of molecular dynamics time units.

and for the strange and charm quark masses:

$$
\operatorname{am}_{q}(\beta)=\frac{c_{q}^{(0)} f(\beta)+c_{q}^{(2)}(10 / \beta) f^{3}(\beta)}{1+d_{q}^{(2)}(10 / \beta) f^{2}(\beta)}\left(\frac{20 b_{0}}{\beta}\right)^{4 / 9}
$$

where $q=s, c$ and

$$
f(\beta)=\left(\frac{10 b_{0}}{\beta}\right)^{-b_{1} /\left(2 b_{0}^{2}\right)} \exp \left(-\beta / 20 b_{0}\right)
$$

is the perturbative two-loop $\beta$-function for three flavors. We checked that using the four-flavor $\beta$-function renormalizes the coefficients but produces the same (within the numerical accuracy) results for the scale and quark masses.

The parameters and accumulated statistics for the zero-temperature ensembles are shown in Table 1. The entries marked with asterisk are used to set the LCP and lattice scale. Corresponding temperatures and the statistics for the finite-temperature ensembles are shown in Table 2 .

\section{Trace anomaly}

At lattice spacing $a$ the trace of the energy-momentum tensor, or interaction measure, can be 


\begin{tabular}{|l|l|r|l|r|r|r|}
\hline & \multicolumn{2}{|c|}{$N_{\tau}=6$} & \multicolumn{2}{c|}{$N_{\tau}=8$} & \multicolumn{2}{c|}{$N_{\tau}=10$} \\
\hline$\beta$ & $T$ & $\mathrm{TU} / 10^{3}$ & $T$ & $\mathrm{TU} / 10^{3}$ & $T$ & $\mathrm{TU} / 10^{3}$ \\
\hline 5.400 & 149 & 10 & & & & \\
5.469 & 160 & 34 & & & & \\
5.541 & 171 & 34 & & & & \\
5.600 & 182 & 10 & 136 & 20 & & \\
5.663 & 193 & 34 & 145 & 30 & & \\
5.732 & 207 & 30 & 155 & 42 & & \\
5.800 & 218 & 10 & 163 & 10 & 131 & 40 \\
5.855 & 235 & 30 & 176 & 45 & 140 & 42 \\
5.925 & 253 & 30 & 190 & 45 & 152 & 42 \\
6.000 & 272 & 10 & 204 & 10 & 163 & 40 \\
6.060 & 291 & 30 & 218 & 39 & 175 & 42 \\
6.122 & 310 & 30 & 233 & 39 & 186 & 42 \\
6.180 & & & 247 & 11 & 197 & 40 \\
6.238 & & & & & 210 & 14 \\
6.300 & 369 & 10 & 277 & 10 & 222 & 10 \\
6.530 & & & 352 & 11 & 282 & 9 \\
6.720 & 567 & 10 & 425 & 10 & 340 & 10 \\
7.000 & & & 548 & 8 & 438 & 20 \\
7.140 & 843 & 7 & 632 & 11 & 506 & 19 \\
7.285 & 967 & 3 & 725 & 11 & 580 & 17 \\
\hline
\end{tabular}

Table 2: Inverse gauge coupling, temperatures and the statistics in thousands of molecular dynamics time units for the HISQ finite-temperature ensembles along the $m_{l}=m_{s} / 5 \mathrm{LCP}$.

related to the partition function as

$$
\varepsilon-3 p=-\frac{T}{V} \frac{d \ln Z}{d \ln a}, \quad Z=\int D U D \bar{\psi} D \psi \exp \left(-S_{g}-S_{f}\right),
$$

where $\varepsilon$ is the energy density and $p$ is the pressure.

To normalize the trace anomaly to zero at zero temperature one can take a difference between the finite- and zero-temperature observables at the same values of the gauge coupling and quark masses, i.e. for an observable $X$ :

$$
\Delta(X)=\langle X\rangle_{\tau}-\langle X\rangle_{0} .
$$

The trace anomaly is then given in terms of the basic observables that enter into the action:

$$
\begin{aligned}
\frac{\varepsilon-3 p}{T^{4}} & =-R_{\beta}(\beta)\left[\Delta\left(S_{g}\right)+R_{u}(\beta) \Delta\left(\frac{d S_{g}}{d u_{0}}\right)\right]+R_{\beta}(\beta) R_{m_{s}}(\beta)\left[2 m_{l} \Delta\left(\bar{\psi}_{l} \psi_{l}\right)+m_{s} \Delta\left(\bar{\psi}_{s} \psi_{s}\right)\right] \\
& +R_{\beta}(\beta) R_{m_{c}}(\beta)\left[m_{c} \Delta\left(\bar{\psi}_{c} \psi_{c}\right)+R_{\mathcal{E}_{N}}(\beta) \Delta\left(\bar{\psi}_{c}\left[\frac{d M_{c}}{d \varepsilon_{N}}\right] \psi_{c}\right)\right] .
\end{aligned}
$$

The change of the lattice spacing and the parameters of the action along the LCP are controlled by 

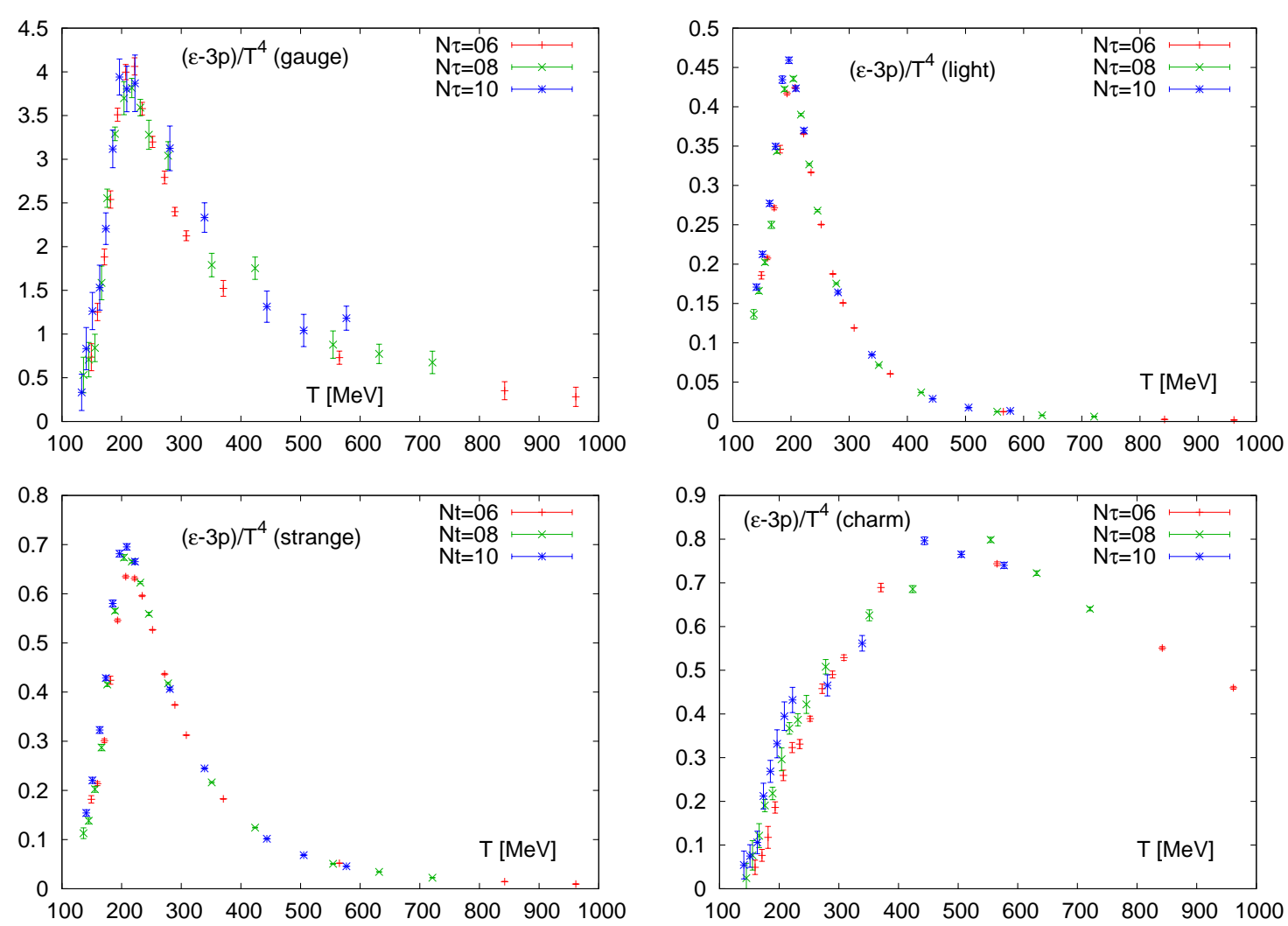

Figure 1: Contributions to the trace anomaly from the gauge field (top left), valence light (top right), strange (bottom left) and charm (bottom right) quarks.

the $\beta$-functions:

$$
\begin{aligned}
R_{\beta}(\beta) & =T \frac{\mathrm{d} \beta}{\mathrm{d} T}=-a \frac{\mathrm{d} \beta}{\mathrm{d} a}=\left(r_{1} / a\right)(\beta)\left(\frac{\mathrm{d}\left(r_{1} / a\right)(\beta)}{\mathrm{d} \beta}\right)^{-1}, \\
R_{m_{q}}(\beta) & =\frac{1}{a m_{q}(\beta)} \frac{\mathrm{d} a m_{q}(\beta)}{\mathrm{d} \beta} \quad \text { for } q=s, c \\
R_{u}(\beta) & =\beta \frac{\mathrm{d} u_{0}(\beta)}{\mathrm{d} \beta} \\
R_{\mathcal{E}}(\beta) & =\frac{\mathrm{d} \varepsilon_{N}(\beta)}{\mathrm{d} \beta}
\end{aligned}
$$

As function of $\beta$ the tadpole factor $u_{0}$ is fit to $u_{0}(\beta)=c_{1}+c_{2} e^{-d_{1} \beta}$, and the Naik term correction $\varepsilon_{N}$ to a polynomial in $\beta$.

The first term in Eq. (3.3) describes the contribution to the trace anomaly from the gauge field, the second from the (valence) light and strange quarks and the third from the (valence) charm quark. These four quantities are shown in Fig. 1. In the peak region around $200 \mathrm{MeV}$ the trace anomaly is dominated by the gauge part. Light and strange quark contributions also reach their maxima around that temperature, while the charm part at $200 \mathrm{MeV}$ contributes less than $10 \%$ to the total result. The charm contribution reaches its maximum around $500 \mathrm{MeV}$ and accounts for $40 \%$ of the value of the trace anomaly at that temperature. (Note, that this is the dominant, valence, 

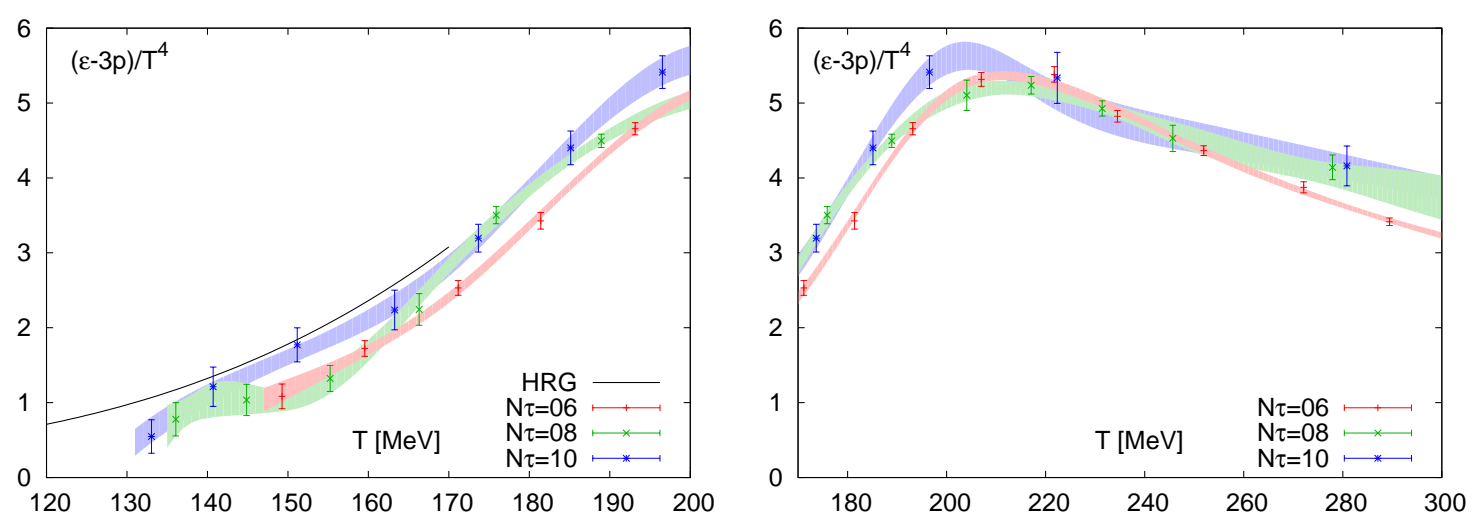

Figure 2: The trace anomaly at low temperatures (left) and in the peak region (right). Bands represent statistical errors on the spline fits.

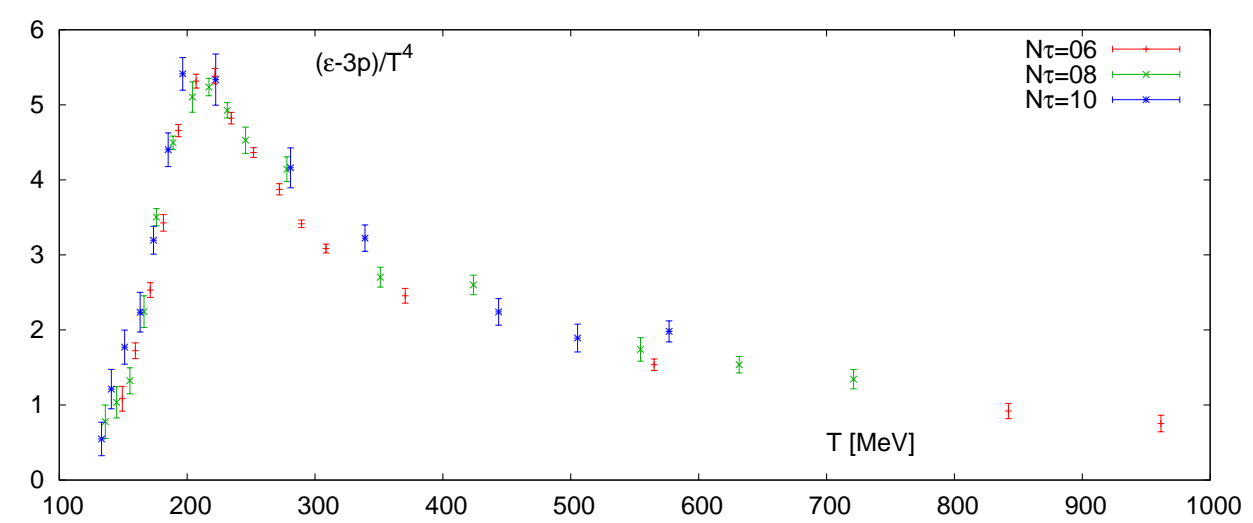

Figure 3: The 2+1+1 flavor trace anomaly with HISQ along the $m_{l}=m_{s} / 5 \mathrm{LCP}$.

charm quark contribution, while the effect of the sea charm can only be quantified by comparing to the $2+1$ flavor equation of state. Such an analysis is left for the future.)

The contribution due to the variation of the charm quark mass in the Naik term, $d M_{c} / d \varepsilon_{N}$ is larger on coarser lattices. We have evaluated it on $N_{\tau}=6$ lattices at $T=272$ and $369 \mathrm{MeV}$, and corresponding zero-temperature ensembles. This quantity is about $2 \%$ of the total result, which is comparable to the statistical errors. Therefore in the present analysis, in particular, for the quantity in Fig. 1 (bottom right) and for the total trace anomaly, we did not include the term $\Delta\left(\bar{\psi}_{c}\left[d M_{c} / d \varepsilon_{N}\right] \psi_{c}\right)$. Its inclusion will presumably decrease the cutoff effects on the charm contribution at low temperatures, $150-200 \mathrm{MeV}$.

We fitted the data for the trace anomaly with splines, shown as bands in Fig. 2. The width of the bands represents only the statistical error, estimated by bootstrap. In Fig. 目 (left) the lowtemperature region is shown. The solid line is the hadron resonance gas (HRG) model result. Coarser lattices produce a heavier hadron spectrum, thus, the approach to the continuum is from below (at least, when the temperature is set with the $r_{1}$ scale). In the peak region, Fig. 2(right), the cutoff effects are mild, and at temperatures around $200 \mathrm{MeV}$ and above, where the light hadrons melt, they are presumably not caused by taste symmetry breaking in the quark sector.

The total trace anomaly is shown in Fig. 3. The $N_{\tau}=10$ lattices extend to $580 \mathrm{MeV}$. This is enough to cover the peak of the valence charm contribution, but without further $N_{\tau}=10$ data the 
cutoff effects above this temperature are thus hard to quantify.

\section{Conclusion}

We have extended our calculation of the $2+1+1$ flavor QCD equation of state with highly improved staggered quarks in two ways. We generated several new zero- and finite-temperature ensembles to provide better coverage of temperatures in the range 130-1000 MeV and substantially increased the statistics on most of the finite temperature ensembles. We have reached lattice spacings down to $0.034 \mathrm{fm}$, which corresponds to $967 \mathrm{MeV}$ on the coarsest, $N_{\tau}=6$ lattice. The charm contribution to the trace anomaly becomes non-negligible around $300 \mathrm{MeV}$ and reaches the maximum far in the deconfined phase, around $500 \mathrm{MeV}$. The cutoff effects on the trace anomaly are significant at low temperatures and are mild in the peak region. However, $N_{\tau}=12$ ensembles will be needed for a reliable continuum extrapolation.

\section{Acknowledgments}

This work was supported by the U.S. Department of Energy and National Science Foundation. Computations for this work were done at the Argonne Leadership Computing Facility (ALCF), the National Center for Atmospheric Research (UCAR), Bluewaters at the National Center for Supercomputing Resources (NCSA), the National Energy Resources Supercomputing Center (NERSC), the National Institute for Computational Sciences (NICS), the Texas Advanced Computing Center (TACC), and the USQCD facilities at Fermilab, under grants from the NSF and DOE.

\section{References}

[1] A. Bazavov et al. [HotQCD], Phys. Rev. D 80, 014504 (2009) [arXiv:0903.4379 [hep-lat]]; A. Bazavov [HotQCD], Nucl. Phys. A904-905 2013, 877c (2013) [arXiv:1210.6312 [hep-lat]].

[2] Y. Aoki, Z. Fodor, S. D. Katz and K. K. Szabo [Budapest-Wuppertal], JHEP 0601, 089 (2006) [hep-lat/0510084]; S. Borsanyi et al. [Budapest-Wuppertal], JHEP 1011, 077 (2010) [arXiv:1007.2580 [hep-lat]]; S. Borsanyi et al. [Budapest-Wuppertal], arXiv:1309.5258 [hep-lat].

[3] S. Borsanyi et al. [Budapest-Wuppertal], PoS LATTICE 2011, 201 (2011) [arXiv:1204.0995 [hep-lat]]; C. Ratti et al. [Budapest-Wuppertal], Nucl. Phys. A 904-905, 869c (2013).

[4] A. Bazavov et al. [MILC], PoS LATTICE 2012, 071 (2012).

[5] A. Bazavov et al. [HotQCD], Phys. Rev. D 85, 054503 (2012) [arXiv:1111.1710 [hep-lat]].

[6] M. Laine and Y. Schroder, Phys. Rev. D 73, 085009 (2006) [hep-ph/0603048].

[7] M. Cheng [RBC-Bielefeld], PoS LAT 2007, 173 (2007) [arXiv:0710.4357 [hep-lat]]; L. Levkova, PoS LAT 2009, 170 (2009) [arXiv:0910.3006 [hep-lat]]; C. DeTar et al. [MILC], Phys. Rev. D 81, 114504 (2010) [arXiv:1003.5682 [hep-lat]].

[8] A. Bazavov et al. [MILC], Phys. Rev. D 82, 074501 (2010) [arXiv:1004.0342 [hep-lat]]; A. Bazavov et al. [MILC], Phys. Rev. D 87, 054505 (2013) [arXiv:1212.4768 [hep-lat]].

[9] E. Follana et al. [HPQCD/UKQCD], Phys. Rev. D 75, 054502 (2007) [hep-lat/0610092].

[10] R. Sommer, Nucl. Phys. B 411, 839 (1994) [hep-lat/9310022]; C. W. Bernard et al. [MILC], Phys. Rev. D 62, 034503 (2000) [hep-lat/0002028]. 